\title{
Biodiversity maintenance in food webs with regulatory environmental feedbacks
}

\author{
Carey K. Bagdassarian ${ }^{\mathrm{a}, *}$, Amy E. Dunham ${ }^{\mathrm{b}, 1}$, Christopher G. Brown ${ }^{\mathrm{a}, 2}$, Daniel Rauscher ${ }^{\mathrm{a}, 2}$ \\ ${ }^{a}$ Department of Chemistry, College of William and Mary, P.O. Box 8795, Williamsburg, VA 23187-8795, USA \\ ${ }^{\mathrm{b}}$ Department of Organismic and Evolutionary Biology, Harvard University, Harvard University Herbaria, 22 Divinity Avenue, Cambridge, MA 02138, USA
}

Received 14 July 2006; received in revised form 26 November 2006; accepted 11 December 2006

Available online 15 December 2006

\begin{abstract}
Although the food web is one of the most fundamental and oldest concepts in ecology, elucidating the strategies and structures by which natural communities of species persist remains a challenge to empirical and theoretical ecologists. We show that simple regulatory feedbacks between autotrophs and their environment when embedded within complex and realistic food-web models enhance biodiversity. The food webs are generated through the niche-model algorithm and coupled with predator-prey dynamics, with and without environmental feedbacks at the autotroph level. With high probability and especially at lower, more realistic connectance levels, regulatory environmental feedbacks result in fewer species extinctions, that is, in increased species persistence. These same feedback couplings, however, also sensitize food webs to environmental stresses leading to abrupt collapses in biodiversity with increased forcing. Feedback interactions between species and their material environments anchor food-web persistence, adding another dimension to biodiversity conservation. We suggest that the regulatory features of two natural systems, deep-sea tubeworms with their microbial consortia and a soil ecosystem manifesting adaptive homeostatic changes, can be embedded within niche-model food-web dynamics.
\end{abstract}

(C) 2007 Elsevier Ltd. All rights reserved.

Keywords: Environmental feedbacks; Food webs; Predator-prey dynamics; Niche model; Species persistence

\section{Introduction}

Organisms within an ecosystem are constantly interacting with and altering their abiotic environment. While classic food-web studies have focused mainly on trophic interactions and energy fluxes (Elton, 1927; Lindeman, 1942) unraveling the effects of environmental feedbacks and environmental modification by organisms on biodiversity maintenance is a current focus in ecological research (de Ruiter et al., 2005; Harding, 1999; Hooper et al., 2005; Loladze et al., 2004; Wright and Jones, 2006; Wright et al., 2006). We propose that organismal feedback-coupled interactions with the environment are likely critical components both for sustaining biodiversity in complex,

\footnotetext{
*Corresponding author. Tel.: + 1757221 2556; fax: + 17572212715.

E-mail address: ckbagd@wm.edu (C.K. Bagdassarian).

${ }^{1}$ Present address: Monitoring and Assessment of Biodiversity Program, National Zoological Park, Smithsonian Institution, 1100 Jefferson Drive, SW, Suite 3123, Washington DC 20560, USA.

${ }^{2}$ These authors contributed equally to this work.
}

multiple trophic level food webs and for altering their susceptibility to environmental stressors.

Intuitive and empirical conclusions that complexity is a characteristic feature of natural communities of species stand at odds with May's theoretical demonstration that increasing biodiversity and complexity destabilize food webs (May, 1971, 1973; and see DeAngelis, 1975; de Ruiter et al., 1995; Hutchinson, 1959; MacArthur, 1955; Neutel et al., 2002; Odum, 1953; Worm and Duffy, 2003; Yodzis, 1981). Current stability-complexity studies continue to be based upon model food webs with diverse predator-prey links or numbers of species. For these food-web structures, predator-prey dynamics are described through increasingly sophisticated systems of differential equations motivated by Lotka-Volterra-like formulations (Brose et al., 2003; Chen and Cohen, 2001; Kondoh, 2003; McCann, 2000). However, these systems are typically prone to significant numbers of species extinctions. Fundamental understanding of the mechanisms - and often identification of the mechanisms - ensuring food-web integrity remains a 
challenge, and several important schemes leading to increased species persistence have been proposed.

For example, different forms for predator-prey functional responses that alter consumption rates of abundant or rare food resources have successfully been used to promote persistence of large fractions of species in large food webs (Martinez et al., 2006; Williams and Martinez, 2004). Complementary to these studies of large food-web dynamics are explorations addressing the effects of removal of keystone species in increasingly complex foodweb structures (Brose et al., 2005). In a different vein, adaptive foraging strategies have been introduced into food-web dynamics whereby predators continuously modify their consumption efforts to focus on those prey yielding above-average energy gains (Brose et al., 2003; Kondoh, 2003, 2006). These strategies are found to increase species persistence in large food webs and to potentially reverse the complexity-stability relationship so that more complex food webs are more stable. Evolutionary schemes in which food-web construction proceeds through sequential species addition leading either to new and persistent predator-prey interactions or to extinction events have been used to create complex food webs with realistic trophic structuring (Drossel et al., 2001; Loeuille and Loreau, 2005; McKane, 2004). Other studies aimed at understanding dynamical food-web persistence have drawn attention to predator-prey body size effects (Emmerson and Raffaelli, 2004) and to coupled fast and slow energy channels for production-to-biomass ratios that exist in natural food webs (Rooney et al., 2006). This latter study connects to earlier work where weak-link interactions between species are shown to promote community persistence (McCann et al., 1998) and resonates with a recent study revealing asymmetric interactions between mutually dependent animal and plant species as an important key to biodiversity maintenance in real food webs (Bascompte et al., 2006). Furthermore, non-dynamical simulations of species removal and subsequent secondary extinctions based upon structural considerations in well-characterized real food webs have contributed to understanding stability-complexity relationships (Dunne et al., 2002).

None of these food-web models, however, take into consideration feedbacks and couplings between organisms and their environment. However, ecosystem function beyond the "who-eats-whom" of food-web dynamics is important to food-web studies (Moorcroft, 2003; Pascual, 2005) and potentially, as in the context of our work, to species persistence. While studies including biogeochemical considerations and ecological stoichiometric constraints (DeAngelis, 1992; Elser et al., 1998; Moe et al., 2005; Schlesinger, 1997; Sterner and Elser, 2002) do place organisms in dynamic physical-chemical environments, effects on multiple trophic level food-web dynamics are not typically addressed: small, often analytically tractable, systems are in focus (Daufresne and Loreau, 2001a, b; Grover, 2003; Hall et al., 2006; Kuijper et al., 2004; Loladze et al., 2004). We still lack a basic understanding of how environmental feedbacks impact the dynamics and persistence of structurally realistic food webs. The work presented here is a contribution in that direction.

The environmental feedbacks we consider are regulatory in nature. That is, within a food web a subset of species regulates an environmental variable through feedback couplings with it. Regulatory feedbacks add functional complexity to food-web models, thereby introducing a new dimension to the stability-complexity debate.

Specifically, we have extended food-web models constructed through the niche model (Williams and Martinez, 2000) - which results in realistic structural characteristics including hierarchical trophic structure, cannibalism, and looping - to include simple regulatory environmental feedbacks in the form of Lovelock's Daisyworld (DW) (Lovelock, 1992; Watson and Lovelock, 1983). Although a theoretical construct, DW's elegant simplicity and wellunderstood mathematical structure make it an excellent candidate for studying species persistence in food webs coupled to environmental feedbacks.

In the original DW system, two daisy species, white and black, populate the surface of a virtual planet. White daisies reflect sunlight and so cool their local environment, while black daisies absorb sunlight and cause warming. These environmental feedbacks result in a self-organized ratio of black to white daisies for temperature regulation over a wide range of the sun's luminosity. It is in this sense that we refer to "regulatory environmental feedbacks." In our food webs, the autotrophs are modeled upon the daisies of DW for their environmental coupling.

By generating food webs with the niche-model algorithm (Williams and Martinez, 2000), we are able to study the effects of environmental feedbacks on a multitude of realistic and structurally diverse food webs. To our knowledge only two previous studies consider DW function in a food-web context (Harding, 1999; Lovelock, 1992). Unlike these studies, we examine the effects of environmental feedbacks on the extinction probability of species within systems featuring high trophic diversity, realistic structural characteristics, and multi-species predator-prey dynamics.

Two natural systems may feature comparable regulatory mechanisms. Hunt and Wall report that the adaptive homeostatic changes they observe in their study of carbon and nitrogen transfers in a soil ecosystem are "reminiscent" of DW (Hunt and Wall, 2002). Furthermore, mutualistic interactions between deep-sea tubeworms $L$. luymesi, their symbionts and external microbial consortia create a feedback system allowing for emergent matching of sulfide supply and demand to the tubeworms (Cordes et al., 2005). We propose that the regulatory features of these systems can also be embedded within niche-model food webs.

\section{Methods}

The focus of this paper is species persistence in food webs with environmental feedbacks: after simulation of 
predator-prey dynamics, species persistence is reported specifically as the ratio of the number of surviving species to the starting number in a given web.

Collections of 10-, 15-, and 20-species food webs were constructed through the niche-model algorithm developed by Williams and Martinez (2000). Briefly, in order to generate an $N$-species food web each species is assigned a random niche value and consumes all others with niche values falling within a feeding range centered at a value below (and up to) the consumer's. More specifically, the niche value $n_{i}$ for species $i$ drawn from a uniform distribution $[0,1]$ and the quantity $x_{i}$ drawn randomly from a beta-probability distribution $p(x)=\beta(1-x)^{\beta-1}$ for $0<x<1$ together define the feeding range for species $i$ as $n_{i} x_{i}$. The center of this feeding range is placed randomly and uniformly in the interval $\left[n_{i} x_{i} / 2, n_{i}\right]$. Species $i$ eats all other species whose niche values fall within $i$ 's feeding range. The connectance $C$ of a food web is defined as $L / N^{2}$, with $L$ being the number of actual predator-prey links in an $N$-species web and $N^{2}$ the total number of possible links. The parameter $\beta$ affects the connectance of niche-model food webs: increasing $\beta$ values are correlated with decreases in $C$. Application of the niche model results in food webs with realistic structures including cannibalism and looping (Williams and Martinez, 2000).

In our work, a potential food web is discarded if (a) all species are not ultimately linked, that is, if any two distal species are not connected via trophic links through other species, (b) two species share the same predators and prey (the focus is on trophic species as in Williams and Martinez), (c) mutually predatory species have no other prey and (d) cannibals have no other resources. Finally, any food web that does not contain exactly two autotrophs at the lowest trophic level is also discarded. Food webs are assigned to separate bins according to their connectance value $C$, with each bin holding 400 unique food webs. For the 10-species study, all food webs within a given bin have exactly the same $C$-value with $C$ increasing in increments of 0.01 in each successive bin. The range of $C$ from 0.10 (the lower bound for 10 -species webs) to 0.35 is examined. 20 species food webs are binned from $C=0.07$ to $C=0.35$; however, the $C=0.09 \mathrm{bin}$, for example, contains webs with connectance in the range $0.0875-0.0925$. Although food webs with connectance values of 0.05 and 0.06 are possible for 20 species, they are difficult to generate through the niche-model algorithm given the retention criteria above and constraint to two autotrophs. The range $C=0.07$ to $C=0.35$ is representative of six well-documented natural food webs (Cattin et al., 2004; Williams and Martinez, 2000). With these constructions, food-web dynamics can be studied as a function of connectance as is usual in stability-complexity considerations.

In each food web, the two autotrophs are indirectly coupled to each other through environmental feedbacks: They are designated as the daisies of Daisyworld (Lovelock, 1992; Watson and Lovelock, 1983). Through the system of equations below, the autotrophs regulate global temperature to values commensurate with their maximal growth rate, even while being consumed by herbivores. Specifically, though predator-prey dynamics disrupt DW steady-state attractors, the autotrophic daisies nonetheless retain their regulatory capacities even when embedded in food webs. Fig. 1 highlights a typical (10species) niche-model food web where two autotrophic daisies are coupled to environmental temperature.

Food-web predator-prey dynamics are based upon bioenergetic consumer-resource models, developed initially by Yodzis and coworkers and generalized by Williams and Martinez (Brose et al., 2003; Martinez et al., 2006; McCann et al., 1998; McCann and Yodzis, 1994a,b; Williams and Martinez, 2004; Yodzis and Innes, 1992). To capture food-web dynamics with environmental feedbacks, we merge these models with Daisyworld equations modified to express autotroph populations as biomasses. For $N$-species, the biomass $B_{i}$ of species $i$ changes with time as

$$
\begin{aligned}
\frac{\mathrm{d} B_{i}}{\mathrm{~d} t}= & r_{i} B_{i} g_{i}\left[1-\frac{\left(B_{1}+B_{2}\right)}{K}\right]-x_{i} B_{i} \\
& +\sum_{j=1}^{N}\left[x_{i} y_{i j} \alpha_{i j} F_{i j}(\bar{B}) B_{i}-x_{j} y_{j i} \alpha_{j i} F_{j i}(\bar{B}) B_{j}\right] .
\end{aligned}
$$

Only the autotrophs $(i=1,2)$ feature an intrinsic growth term: $r_{1}=r_{2}=1$ for them and $r_{i}=0$ for all other species. Autotroph biomasses, $B_{1}$ and $B_{2}$, are constrained to a combined total value of 2 by the carrying capacity, $K=2$. Furthermore, autotroph growth rate is modulated by $g_{i}$ $(i=1,2)$ which couples the system to Daisyworld environmental feedbacks (see below). The mass-specific metabolic rate $x_{i}$ is 0.3 for the autotrophs and 0.2 otherwise. The maximum rate (per unit metabolic rate of $i$ ) at which species $i$ consumes $j$ is $y_{i j}=3.5$. The term $\alpha_{i j}$ is derived from the connectivity matrix $C_{i j}$ as $\alpha_{i j}=C_{i j} / \sum_{j} C_{i j}$ where $C_{i j}=1$ if species $i$ consumes $j$, and is 0 otherwise.

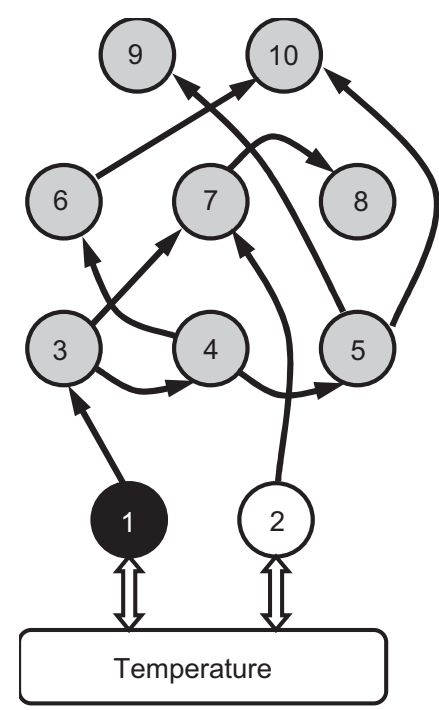

Fig. 1. Representative 10-species niche-model food web with connectance 0.10 . Black and white autotrophs are shown with feedback couplings to temperature. 
Clearly, $\alpha_{i j}$ is normalized so that $\sum_{j} \alpha_{i j}=1$ and reflects a species' relative preference for a particular resource; in this work a consumer uses equally all its resources. Also, $\alpha_{1 j}=\alpha_{2 j}=0$ for all $j$ since species 1 and 2 are the autotrophs. The functional response is that of Brose et al. (2003) modeled after Holling's Type II expression (Holling, 1965):

$F_{i j}(\bar{B})=\frac{B_{j}}{\sum_{k=1}^{N} \alpha_{i k} B_{k}+B_{0}}$,

where the half-saturation biomass $B_{0}=0.3$. A species is considered extinct if its biomass falls below $10^{-15}$ during simulation of food-web predator-prey dynamics. The term $\alpha_{i j}$ is recalculated after every species extinction: if species $j$ goes extinct, $C_{i j}$ is changed from 1 to 0 if species $i$ previously preyed upon it.

Our environmental feedbacks, adopted from Lovelock's Daisyworld (Lovelock, 1992; Watson and Lovelock, 1983), are as follows and the reader is referred to the original literature for details. The first autotroph (species 1) absorbs sunlight and heats its local environment; species 2 reflects sunlight and so cools its environment. This difference between autotrophs (the two daisy species) is captured by their albedos or reflectivities, $A_{1}=0.15$ and $A_{2}=0.85$ for the black and white autotrophs, respectively, and leads to global temperature regulation. That is, for a wide range of the sun's luminosity the system's global temperature is kept nearly constant through a self-organized ratio of the twoautotroph types. An abiotic system, on the other hand, shows a monotonic increase in temperature with increasing luminosity. In keeping with the original DW model, bare ground without autotroph cover is assigned an albedo $A_{g}=0.5$. The overall average albedo is modified to reflect autotroph biomasses,

$A=A_{g}\left[1-\frac{B_{1}+B_{2}}{K}\right]+\frac{A_{1} B_{1}+A_{2} B_{2}}{K}$.

The global temperature is Watson and Lovelock's

$T=(S L(1-A) / \sigma)^{1 / 4}-273$,

with $L$ the relative solar luminosity, $S=917 \mathrm{~J} \mathrm{~m}^{-2} \mathrm{~s}^{-1}$ (stellar constant), and $\sigma=5.669 \times 10^{-8} \mathrm{~J} \mathrm{~K}^{-4} \mathrm{~m}^{-2} \mathrm{~s}^{-1}$ (Stefan-Boltzmann constant). In the predator-prey dynamics model above, $g_{i}(i=1,2)$ couples autotroph growth rates to their local temperatures $T_{i}$ :

$g_{i}=1-\gamma\left(22.5-T_{i}\right)^{2}$.

Local autotroph temperatures are the same as Watson and Lovelock's:

$T_{i}=20\left(A-A_{i}\right)+T$.

When $\gamma=0.00326$, the value used in the original DW work, food-web dynamics are coupled to environmental feedbacks. If $\gamma=0$, however, all environmental feedbacks and temperature regulation are turned off and the system reduces to a conventional food web. Consequently, foodweb dynamics can be simulated with or without environ- mental coupling for each trophic structure. Unless stated otherwise, the sun's luminosity is tuned so that autotroph biomasses are equal when no overlying food-web structure exists. This involves simply solving for $L$ in Eq. (4) when the average albedo $A=0.5$ with the global temperature set at $22.5^{\circ} \mathrm{C}$. Since the feedbacks regulate global temperature in the neighborhood of $22.5^{\circ} \mathrm{C}$ through Eq. (5), choosing this temperature in Eq. (4) ensures minimal "functional stresses" upon the autotrophs. The resulting luminosity is $L=0.94$.

For each food-web structure, with and without environmental feedbacks, the persistent species are those whose biomasses do not fall below $10^{-15}$ during simulation of predator-prey dynamics. Each species' initial biomass is chosen randomly from a value between $10^{-15}$ and 1 . Predator-prey dynamics for 10 -species food webs are simulated for 2500 time steps, while 20 -species webs run for 4000 time steps. The number of species extinctions for each web is tallied after a dynamical run and species persistence is reported as the ratio of the number of surviving species to the starting number $(10,15$, or 20 , depending on web size). Though species persistence for any given food web is somewhat sensitive to initial species biomasses, overall conclusions are qualitatively and nearly quantitatively the same with or without averaging over different initial conditions.

\section{Results and analysis}

The number of species extinctions and, consequently, species persistence are calculated for every food web at each connectance value, with and without environmental feedbacks, that is, with the parameter $\gamma=0.00326$ and with $\gamma=0$. Species persistence, again with and without regulatory feedbacks, is averaged over all food webs at a given connectance. We find that for food webs at each level of biodiversity (10-, 15-, and 20-species) average species persistence increases when temperature feedbacks are turned on regardless of food-web connectance. Results for 10- and 20-species niche-model food webs are shown in Fig. 2(A) and (B), respectively, and the 15-species webs have qualitatively similar increases in species persistence in the presence of feedback regulation. DW environmental feedback regulation promotes food-web persistence, with its effects on species persistence declining (especially for 20species webs) with increasing connectance, as is clear from Fig. 2(A) and (B). For all common connectance values $(0.10-0.35)$, the percent increase in species persistence is greater for the 10-species food webs (although at $C=0.10$ both 10 - and 20 -species webs show a similar increase in persistence of approximately 32\%). The results and analysis below focus on the 10 -species webs.

Note that our expression for autotroph growth rate (first term in Eq. (1)) differs from that used by Brose et al. (2003) and Kondoh (2006). This leads to a species persistence vs. connectance relation for the 10 -species webs that does not decrease strictly monotonically when environmental 


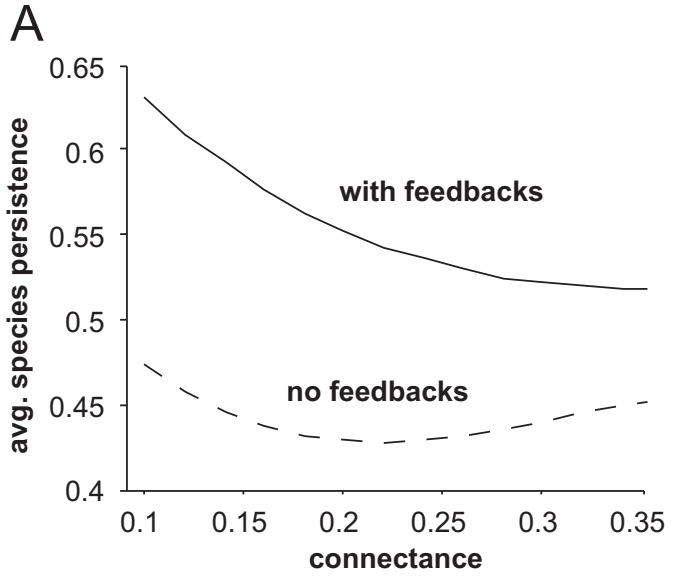

B

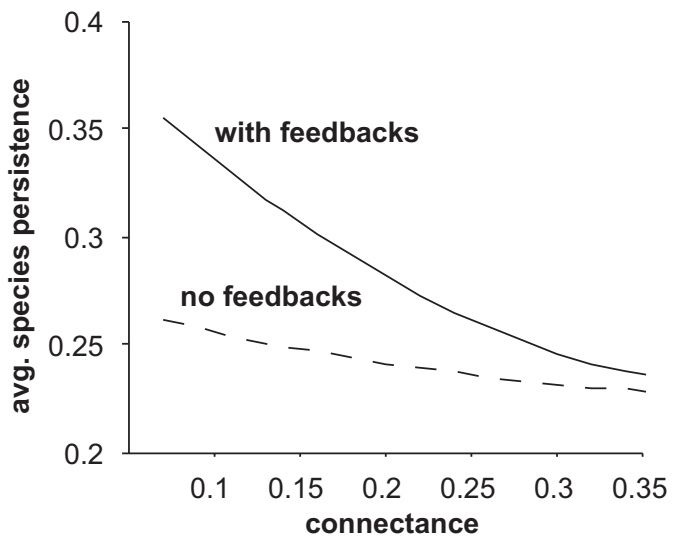

Fig. 2. (A) Results for 10-species food webs. Environmental feedbacks lead to increased average species persistence through the entire connectance range. (B) Results for 20-species food webs. For both 10- and 20species webs the mass-specific metabolic rate $x_{i}=0.3$ for autotrophs and 0.2 otherwise, the maximum rate at which species $i$ consumes $j$ is $y_{i j}=3.5$, luminosity is tuned to 0.94 , and DW coupling $\gamma=0.00326$. Moderate deviations from the luminosity value $( \pm 0.06)$ do not affect the results. Polynomial curve fit to data sets.

feedbacks are off, that is, when conventional food-web dynamics are considered (see bottom curve of Fig. 2(A)). Switching to the Brose et al. and Kondoh expression-to $r_{i} B_{i}\left(1-B_{i} / K\right)$ with $i=1,2$ - does give our 2-autotroph food webs a monotonic decrease in persistence with increasing connectance in the absence of DW coupling, consistent with the case where Brose et al. and Kondoh address conventional food-web dynamics. Our expression, however, allows for a natural coupling to Daisyworld dynamics.

Fig. 3 is constructed from the 40010 -species food webs having a connectance value of 0.12 and for which feedback-induced increases in species persistence are quite pronounced. Plotted on the $y$-axis is the fraction of the total number of food webs with a given number of extinctions. Here, food-web dynamics are simulated for each web from a single set of (randomly distributed) initial species biomasses: no averages over different sets of initial biomasses are performed and, consequently, the number of

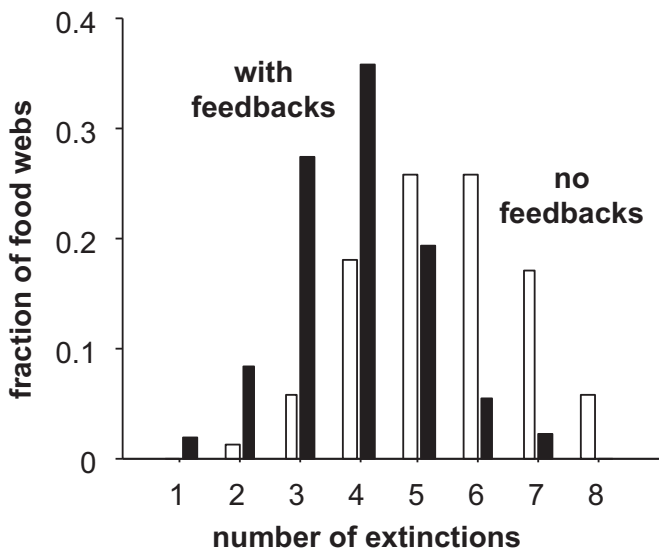

Fig. 3. With environmental feedbacks, the distribution of species extinctions shifts to the left to peak at smaller numbers. The maximum number of extinctions for feedback-regulated webs is 7 species, while the minimum number in the unregulated case is found to be 2 . Results are for 10 -species food webs with connectance $C=0.12$ and with parameters as in Fig. 2. Feedback scenario represented by black-fill bars; no feedback scenario represented by white-fill bars.

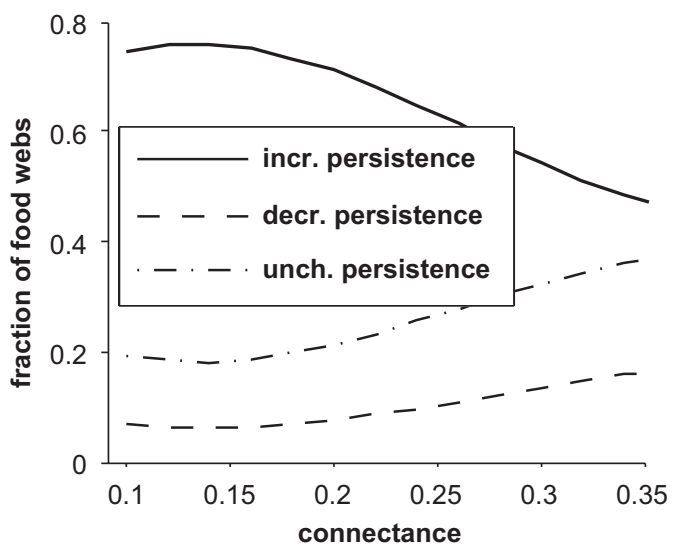

Fig. 4. Fraction of food webs showing increased, decreased, or unchanged persistence when environmental feedbacks are on. Results for 10-species food webs with parameters as in Fig. 2 .

extinctions per web, either with or without feedback regulation, is a whole number. The shift with feedback regulation to fewer species extinctions is apparent. Similar results hold for other connectance values and for higher species numbers, though with less demarcation between the regulated and unregulated scenarios at the highest $C$ values.

Further examination shows that while environmental feedbacks at the chosen luminosity lead to increased species persistence for most food webs, in others environmental coupling results in decreased or unaffected persistence. At each connectance value, we can calculate the fractions of food webs that show increased, decreased, and unaffected species persistence when environmental feedbacks are turned on. As seen in Fig. 4 (for 10-species webs), the fraction of food webs with feedback-induced increases in persistence declines with increasing connectance, and the 
fraction of food webs with decreased or unaffected persistence increases. Furthermore, those food webs for which environmental feedbacks lead to increased persistence have, on average, approximately two less species extinctions per web while decreased-persistence food-web networks have between 1 and 1.5 more extinction events. This result together with Fig. 4 explains the trend shown in Fig. 2(A) where, on average, feedback-induced increases in persistence are greater at lower connectance values. For food webs with connectance 0.12 , the maximum feedbackinduced decrease in persistence is an additional three extinctions per web, while maximum feedback-stabilization involves 6 fewer species extinctions. Furthermore, 30\% of all food webs with increased persistence at this connectance gain three or more persistent species over the feedback-free case and 3\% show a dramatic increase by five or more species.

All results are qualitatively similar for 10-, 15- and 20 -species webs, though the average number of extinctions, with or without feedback regulation, increases with food web size. Results are robust to changes in the feeding rate of predators on their resources: predator-prey dynamics with $y_{i j}$ values (see Eq. (1)) in the range tested (1.5-7.5) show qualitatively similar increases in species persistence when environmental feedbacks are active. Either weakening or strengthening the coupling between food-web and environmental-feedback dynamics by decreasing $\gamma$ from 0.00326 to 0.0015 or by increasing it to 0.0065 (with all other parameter values as given in Section 2) also leaves the persistence results qualitatively unaltered. The same is true for changing the autotroph to consumer ratio for metabolic rates $x_{i}$ from $0.3: 0.2$ to $0.3: 0.1$ or to $0.3: 0.4$. These results for 10-species food webs are summarized in Fig. 5 where percent increases in species persistence resulting from environmental feedbacks are plotted against connectance for various parameter sets. Note the considerable increase

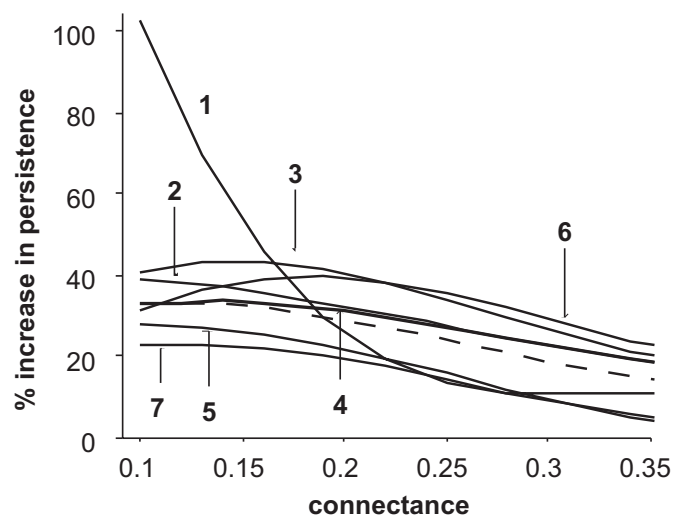

Fig. 5. Percent increase in average species persistence with environmental regulation. Results are for 10 -species food webs with luminosity $L=0.94$. The dashed curve is for $y_{i j}=3.5, \gamma=0.00326$, and a ratio $r$ of metabolic rates $x_{i}$ of 0.3:0.2. Curve 1: $y_{i j}=1.5, \gamma=0.00326, r=0.3: 0.2$. Curve 2: $y_{i j}=5.5, \gamma=0.00326, \quad r=0.3: 0.2$. Curve $3: y_{i j}=7.5, \gamma=0.00326$, $r=0.3: 0.2$. Curve $4: y_{i j}=3.5, \gamma=0.0015, r=0.3: 0.2$. Curve $5: y_{i j}=3.5$, $\gamma=0.0065, r=0.3: 0.2$. Curve 6: $y_{i j}=3.5, \gamma=0.00326, r=0.3: 0.1$. Curve 7: $y_{i j}=3.5, \gamma=0.00326, r=0.3: 0.4$. in persistence at low connectance for the $y_{i j}=1.5$ case. In conclusion, environmental feedbacks foster biodiversity with high probability and especially at lower and more realistic connectance levels.

A mechanistic explanation for the effects of environmental feedbacks on species persistence - why feedbacks lead to both increases and decreases in extinction numbers - is not readily tractable. With feedbacks turned off, predator-prey dynamics lead to one autotroph extinction in $13 \%$ of all 10 -species food webs. Feedbacks ensure autotroph survival in each of these: the demand for temperature regulation imposes restorative forces on autotroph biomass. However, environmental feedbacks, while sustaining both autotrophs in this subset of food webs, lead to overall increases in species persistence in only $69 \%$ of these food webs. Of the remaining, $22 \%$ are left unaffected in their numbers of extinctions, while $9 \%$ have a greater number of extinctions. Autotroph survival via feedbacks does not ensure overall food-web persistence.

In the bare DW model, that is, for autotrophic temperature regulation without overlying food-web structure and predator-prey dynamics, both daisies reach a steady-state biomass of $B_{s}=0.64$ for luminosity $L=0.94$. The majority of food webs, on the other hand, show large species biomass fluctuations over time even in the presence of DW temperature regulation. Let $\left\langle B_{i}\right\rangle$ express the timeaveraged biomass of an autotroph $(i=1,2)$ within a nichemodel food web, with the average taken after transient fluctuations have settled. The two scenarios, predator-prey dynamics with and without feedback regulation, result in different values for $\left\langle B_{i}\right\rangle$-designated as $\left\langle B_{i}\right\rangle_{r}$ and $\left\langle B_{i}\right\rangle_{u}$, respectively - and these values differ also from the steady-state DW-only autotroph biomass $B_{s}$. Consequently, two distance measures $D_{r}=\sqrt{\sum_{i=1}^{2}\left(\left\langle B_{i}\right\rangle_{r}-B_{S}\right)^{2}}$ and $D_{u}=\sqrt{\sum_{i=1}^{2}\left(\left\langle B_{i}\right\rangle_{u}-B_{s}\right)^{2}}$ for each food web can be calculated, thus quantifying the degree to which average autotroph biomass is perturbed away from $B_{s}$ by food-web dynamics with or without feedback regulation, respectively. The distance measures are roughly correlated with the effects of environmental feedbacks on species persistence: When feedback regulation results in increased species persistence in any given food web, most likely $D_{r}<D_{u}$ for that web. That is, for those food webs showing increased persistence with environmental feedbacks, average autotroph biomass is forced away from $B_{s}$ by predator-prey dynamics to a lesser extent when feedbacks are turned on. Specifically, $D_{r}<D_{u}$ for $80 \%$ of all 10 -species food webs that show feedback-induced increased species persistence. On the other hand, $D_{r}<D_{u}$ for $67 \%$ of those food webs where feedback regulation leaves species persistence unaffected and for only $57 \%$ of food webs suffering increased extinctions. Consequently, the tendency to overwhelm the steady-state DW attractors is greatest when environmental feedbacks lead to greater species extinctions.

In all three cases, nonetheless-for increased, decreased, and unaffected persistence-feedbacks result in decreased 
distance to the DW attractor for the majority of food webs and thereby in a lessening of consumers' effects on mean autotroph biomass. As a corollary, it is possible that the dampened impact of consumers on autotroph resource biomass via environmental regulation correlates with fewer extinctions. (We thank an anonymous reviewer for this observation.)

With $\delta B_{i}=\sigma_{B_{i}} /\left\langle B_{i}\right\rangle$ defining relative autotroph biomass fluctuation $\left(\sigma_{B i}\right.$ is the standard deviation in biomass fluctuations for autotroph $i$ ), the subset of food webs where environmental feedbacks decrease both $\delta B_{i}$ and the distance measure $\left(D_{r}<D_{u}\right)$ can be identified. Even in this subset, environmental feedbacks can increase, decrease, or leave unaffected species persistence. We conclude that the effects of environmental feedbacks on species persistence emerge from a food web's entire trophic structure and overlying nonlinear dynamics: The key cannot rest solely on autotroph behavior.

Environmental feedbacks coupled to food-web dynamics introduce an interesting and important sensitivity to environmental stressors. Without overlying food-web structures - that is for the bare DW model-autotroph biomasses are equal when the luminosity is tuned to $L=0.94$. The global temperature is $22.5^{\circ} \mathrm{C}$ at this luminosity, corresponding to a maximal growth rate for both autotrophs (see Eq. (5)) and minimal environmental stress upon them. An environmentally coupled food web can be stressed by forcing the luminosity away from 0.94 . Fig. 6(A) shows the number of species extinctions as a function of luminosity for a representative 10 -species food web (in which environmental feedbacks lead to increased persistence when $L=0.94$ ). The numbers of extinctions reported are averages over different initial conditions for biomass trajectories; that is, the system of differential equations describing predator-prey interactions are integrated from different starting biomasses and the average number of extinctions is calculated. Results without feedbacks therefore simply gauge the mild sensitivity to initial biomass. While feedback coupling enhances biodiversity over a range of the sun's luminosity, catastrophic species extinctions become inevitable as luminosity is forced away from the value corresponding to maximal species persistence. Importantly, biodiversity collapse occurs even though autotroph biomasses remain at potentially healthy levels, as can be seen from the bottom two curves of Fig. 6(A), which show white and black daisy biomasses in the absence of the overlying food web. The autotroph resources for the food web are there, but the coupling between food-web dynamics and environmental feedbacks renders the system vulnerable to environmental forcing. These results hold in general for those food webs with increased species persistence for some luminosity range under feedback regulation.

In Fig. 6(A) the "no feedback" curve represents the unregulated scenario with $\gamma=0$ so that conventional foodweb dynamics apply (see Eq. (5)). The autotrophs neither regulate temperature nor are their growth rates affected by
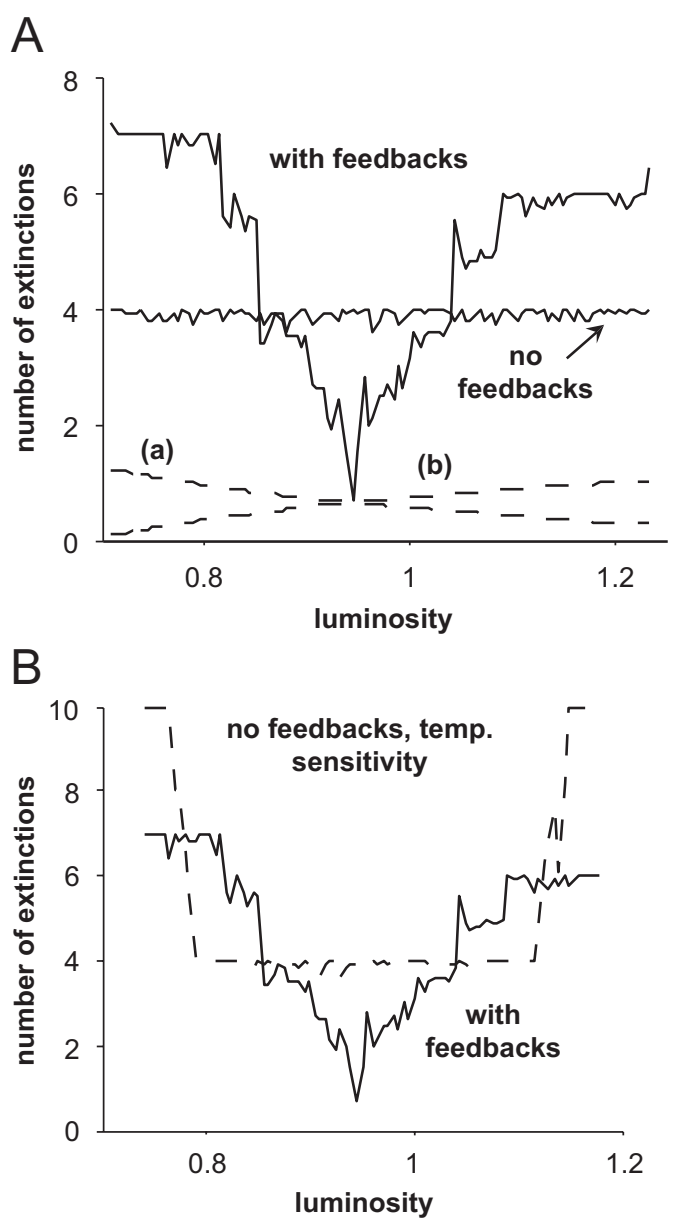

Fig. 6. (A) Extinction results as a function of luminosity, with and without environmental-feedback coupling (top two curves), for a representative 10-species food web with connectance 0.12. Autotroph biomass curves (bottom two; curve (a) is for black daisies, curve (b) is white daisies) reveal DW attractors when modeling only the two autotrophs without overlying food-web dynamics. (B) Extinction results for the same representative food web as in Fig. 6(A). The bold curve is for the fully environmentally coupled system, while the "no feedbacks, temperature sensitivity" result (dashed curve) is for autotrophs with temperature sensitive growth rates but stripped of any ability to regulate global temperature. Mass-specific metabolic rate $x_{i}=0.3$ for autotrophs and 0.2 otherwise, the maximum consumption rate of species $j$ by $i$ is $y_{i j}=3.5$, and DW coupling $\gamma=0.00326$.

it. As an alternative to this full decoupling, we can allow autotroph growth rate to be temperature sensitive $(\gamma=0.00326)$ while shutting off their ability to regulate global temperature. In Eq. (5), the local temperature $T_{i}$ is replaced by global temperature $T$, which in turn is given by Eq. (4) with the albedo set to that of a gray planet $(A=0.5)$. With increasing luminosity, global temperature increases; autotroph growth rates decrease quadratically from a maximum value of 1 when $L=0.94$. The number of extinctions vs. luminosity curve for the representative food web is shown in Fig. 6(B). For the temperature sensitive but no feedback case, the number of extinctions remains constant for a wide range of luminosity and so for a range 
of autotroph growth rates. Food-web collapse at more extreme values for $L$ occurs because of diminished autotroph resources to feed the web. In the fully regulated case on the other hand (see "with feedbacks" curve in Fig. 6(B)), the abrupt loss of biodiversity occurs at luminosities for which the "no feedbacks, temperature sensitive" system still manifests about 4 extinctions. This enhanced sensitivity - as well as the relative increased persistence imparted at extreme luminosities where the no feedbacks, temperature sensitive system has 10 species extinctions-hallmarks the nonlinear couplings of the feedback system and its effects on food-web dynamics. This also underscores the potency of the fully regulated system to increase food-web species persistence when the autotrophs are both temperature sensitive and coupled to temperature regulation through their feedbacks.

\section{Discussion and conclusions}

Daisyworld, although a theoretical construct, provides a powerful inroad to identifying features leading to species persistence in real food webs. We suggest that food webs featuring realistic environmental couplings with attractor dynamics for an environmental variable will also manifest increased overall species persistence.

In their study of soil biodiversity and ecosystem function, Hunt and Wall (2002) studied carbon and nitrogen transfers among plants, microbes, and soil fauna using well-characterized and detailed trophic relationships in a multi-species food web. This is the system referenced above which the authors claim to exhibit Daisyworld-like regulation. Specifically, the authors write that "model response to elevated $\mathrm{CO}_{2}$ is reminiscent of apparently adaptive homeostatic changes." We suggest that the regulatory subsystem of this soil ecosystem can be embedded within diverse niche-model food webs: It is precisely this kind of realistic homeostatic regulation that we expect to contribute to species persistence. In another study, Cordes and coworkers describe and model the mutualistic interactions between the deep-sea tubeworm L. luymesi, its sulfide-oxidizing symbionts, and external sulfate-reducing bacterial consortia (Cordes et al., 2005). There is a stoichiometric symmetry here: The tubewormsymbiont pair consumes sulfide and releases sulfate and the bacterial constortia consume sulfate and release sulfide. With realistic modeling and empirically chosen parameters, these authors show that a nearly 1:1 ratio is maintained between sulfide supply and demand to and from the tubeworms. Without this mutualism between organisms, environmental sulfide alone cannot meet the tubeworms' long-term growth requirements. The 1:1 supply:demand ratio is established as an emergent property, much like DW's global temperature regulation. Additionally, the resultant sequestering of environmental sulfide by the tubeworm system allows for colonization by other organisms otherwise sensitive to sulfide toxicity. As with the soil ecosystem, the regulatory feedbacks of the tubeworm- symbiont-consortia system can form the base of diverse niche-model food webs whose species persistence can then be studied.

The autotrophs in our DW food-web models are more than ultimate food resources. At their core, the autotrophs are a subset of the food web involved in maintaining homeostatic control of an environmental quantity, in this case the global temperature. The soil biodiversity and tubeworm studies above suggest two natural systems in which subsets of species can possibly lead to overall foodweb persistence through their regulatory function.

As previously stated, two other studies address DW function in a food-web context (Harding, 1999; Lovelock, 1992). Lovelock's work incorporates up to three trophic levels: daisies, herbivores (each of which can eat any daisy type), and carnivores (again, each preying upon any of the herbivores). Environmental feedbacks function at each trophic level. Lovelock does not compare food-web persistence with and without feedback regulation but finds that when the feedback-regulated system has reached steady-state at a given luminosity (after the dynamics have run for many time-steps) only a few species survive. Harding's study (Harding, 1999), in which consumers have temperature-dependent growth rates but no regulatory feedback function, proposes a positive correlation between food-web complexity and stability in terms of resilience (return time to steady-state dynamics) and resistance to perturbation. While Harding does not study food-web dynamics in the absence DW regulation, he finds that for all levels of connectance studied 1 carnivore, 3 herbivores, and numerous daisies types (up to 23 are modeled) can coexist after steady-states are achieved. He concludes that adding carnivores and herbivores to a daisy-only system increases biodiversity by allowing more types of daisies to flourish. In our niche-model food webs with long-time nonlinear predator-prey dynamics (where only autotrophs are coupled to temperature regulation and have temperature sensitivity), feedback regulation also increases the probability of increased biodiversity at all levels of foodweb connectance.

In tuning the parameters built into their functional response functions, Martinez et al. (2006) have found increases in species persistence of approximately $50 \%$ for 30 -species niche-model food webs with connectance 0.15 and no constraints on the number of autotrophs. Kondoh (2006)'s work, in which the number of autotrophs is fixed, reports that adaptive foraging strategies result in stability increases with connectance. While environmental feedbacks in our work do not reverse the persistence vs. connectance curve (persistence decreases with increasing connectance), they do lead to considerable increases in species persistence-indeed, approximately $100 \%$ for at least one parameter set at low connectance values (see Fig. 5).

Our extinctions vs. luminosity results have striking implications for the effects of real environmental perturbations such as nutrient loading or atmospheric change on 
species interactions and food-web persistence. While regulatory feedbacks can lead to increased species persistence in moderately stressed food webs thereby giving them stability to environmental perturbations, our models predict eventual and abrupt biodiversity collapse with increased forcing. The importance of "functional species" such as our autotrophs for biodiversity maintenance is apparent. Their removal, either through natural or anthropogenic effects, deprives the food web not only of resources but also of regulatory mechanisms, which contribute to species persistence.

\section{Acknowledgments}

We thank Neo Martinez for insight into the niche model, Jim Elser for discussions, and Volker Rudolf for a critical reading of the manuscript. We are grateful for thoughtful input and suggestions from three anonymous reviewers. The Jeffress Memorial Trust, The College of William and Mary, Harvard University, and the Howard Hughes Medical Institute extended financial support.

\section{References}

Bascompte, J., Jordano, P., Olesen, J.M., 2006. Asymmetric coevolutionary networks facilitate biodiversity maintenance. Science 312, 431-433.

Brose, U., Williams, R.J., Martinez, N.D., 2003. Comment on foraging adaptation and the relationship between food-web complexity and stability. Science 301, 918b.

Brose, U., Berlow, E.L., Martinez, N.D., 2005. Scaling up keystone effects from simple to complex ecological networks. Ecol. Lett. 8, 1317-1325.

Cattin, M.-F., Bersier, L.-F., Banašek-Richter, C., Baltensperger, R., Gabriel, J.-P., 2004. Phylogenetic constraints and adaptation explain food-web structure. Nature 427, 835-839.

Chen, X., Cohen, J.E., 2001. Global stability, local stability and permanence in model food webs. J. Theor. Biol. 212, 223-235.

Cordes, E.E., Arthur, M.A., Shea, K., Arvidson, R.S., Fisher, C.R., 2005 Modeling the mutualistic interactions between tubeworms and microbial consortia. PLoS Biol. 3, e77.

Daufresne, T., Loreau, M., 2001a. Ecological stoichiometry, primary producer-decomposer interactions, and ecosystem persistence. Ecology 82, 3069-3082.

Daufresne, T., Loreau, M., 2001b. Plant-herbivore interactions and ecological stoichiometry: when do herbivores determine plant nutrient limitation? Ecol. Lett. 4, 196-206.

de Ruiter, P.C., Neutel, A.M., Moore, J.C., 1995. Energetics, patterns of interaction strengths, and stability in real ecosystems. Science 269 , $1257-1260$

de Ruiter, P.C., Wolters, V., Moore, J.C., Winemiller, K.O., 2005. Food web ecology: playing Jenga and beyond. Science 309, 68-71.

DeAngelis, D.L., 1975. Stability and connectance in food web models. Ecology 56, 238-243.

DeAngelis, D.L., 1992. Dynamics of Nutrient Cycling and Food Webs. Chapman \& Hill, London.

Drossel, B., Higgs, P.G., McKane, A.J., 2001. The influence of predator-prey population dynamics on the long-term evolution of food web structure. J. Theor. Biol. 208, 91-107.

Dunne, J.A., Williams, R.J., Martinez, N.D., 2002. Network structure and biodiversity loss in food webs: robustness increases with connectance. Ecol. Lett. 5, 558-567.
Elser, J.J., Chrzanowski, T.H., Sterner, R.W., Mills, K.H., 1998. Stoichiometric constraints on food-web dynamics: a whole-lake experiment on the Canadian shield. Ecosystems 1, 120-136.

Elton, C., 1927. Animal Ecology. Sidgwick \& Jackson, London.

Emmerson, M.C., Raffaelli, D., 2004. Predator-prey body size, interaction strength and the stability of a real food web. J. Anim. Ecol. 73, 399-409.

Grover, J.P., 2003. The impact of variable stoichiometry on predator-prey interactions: a multinutrient approach. Am. Nat. 162, $29-43$.

Hall, S.R., Leibold, M.A., Lytle, D.A., Smith, V.H., 2006. Inedible producers in food webs: controls on stoichiometric food quality and composition of grazers. Am. Nat. 167, 628-637.

Harding, S.P., 1999. Food web complexity enhances community stability and climate regulation in a geophysiological model. Tellus 51B, 815-829.

Holling, C.S., 1965. The functional response of predators to prey density and its role in mimicry and population regulation. Mem. Entomol. Soc. Canada 45, 1-60.

Hooper, D.U., Chapin, F.S., Ewel, J.J., Hector, A., Inchausti, P., Lavorel, S., J. Lawton, J.H., et al., 2005. Effects of biodiversity on ecosystem functioning: a consensus of current knowledge. Ecol. Monogr. 75, 3-35.

Hunt, H.W., Wall, D.H., 2002. Modelling the effects of loss of soil biodiversity on ecosystem function. Global Change Biol. 8, 33-50.

Hutchinson, G.E., 1959. Homage to Santa Rosalia or Why are there so many kinds of animals? Am. Nat. 870, 145-159.

Kondoh, M., 2003. Foraging adaptation and the relationship between food-web complexity and stability. Science 299, 1388-1391.

Kondoh, M., 2006. Does foraging adaptation create the positive complexity-stability relationship in realistic food-web structure? J. Theor. Biol. 238, 646-651.

Kuijper, L.D.J., Kooi, B.W., Anderson, T.R., Kooijman, S.A.L.M., 2004. Stoichiometry and food-chain dynamics. Theor. Popul. Biol. 66, 323-339.

Lindeman, R.L., 1942. The trophic dynamic aspect of ecology. Ecology 23, 399-418.

Loeuille, N., Loreau, M., 2005. Evolutionary emergence of size-structured food webs. Proc. Natl Acad. Sci. USA 102, 5761-5766.

Loladze, I., Kuang, Y., Elser, J.J., Fagan, W.F., 2004. Competition and stoichiometry: coexistence of two predators on one prey. Theor. Popul. Biol. 65, 1-15.

Lovelock, J.E., 1992. A numerical-model for biodiversity. Philos. Trans. R. Soc. London B-Biol. Sci. 338, 383-391.

MacArthur, R.H., 1955. Fluctuations of animal population and a measure of community stability. Ecology 36, 533-536.

Martinez, N.D., Williams, R.J., Dunne, J.A., 2006. Diversity, complexity, and persistence in large model ecosystems. In: Pascual, M., Dunne, J.A. (Eds.), Ecological Networks: Linking Structure to Dynamics in Food-webs. Oxford University Press, Oxford, pp. 163-185.

May, R.M., 1971. Stability in multi-species community models. Math. Biosci. 12, 59-79.

May, R.M., 1973. Stability and Complexity in Model Ecosystems. Princeton University Press, Princeton.

McCann, K., Hastings, A., Huxel, G.R., 1998. Weak trophic interactions and the balance of nature. Nature 395, 794-798.

McCann, K., Yodzis, P., 1994a. Biological conditions for chaos in a threespecies food chain. Ecology 75, 561-564.

McCann, K., Yodzis, P., 1994b. Nonlinear dynamics and population disappearances. Am. Nat. 144, 873-879.

McCann, K.S., 2000. The diversity-stability debate. Nature 405, 228-233.

McKane, A.J., 2004. Evolving complex food webs. Eur. Phys. J. B 38, 287-295.

Moe, S.J., Stelzer, R.S., Forman, M.R., Harpole, W.S., Daufresne, T., Yoshida, T., 2005. Recent advances in ecological stoichiometry: insights for population and community ecology. Oikos 109, 29-39.

Moorcroft, P.R., 2003. Recent advances in ecosystem-atmosphere interactions: an ecological perspective. Proc. R. Soc. B-Biol. Sci. $270,1215-1227$. 
Neutel, A.M., Heesterbeek, J.A.P., de Ruiter, P.C., 2002. Stability in real food webs: weak links in long loops. Science 296, 1120-1123.

Odum, E.P., 1953. Fundamentals of Ecology. Saunders, Philadelphia.

Pascual, M., 2005. Computational ecology: from the complex to the simple and back. PloS Comp. Biol. 1, 101-105.

Rooney, N., McCann, K., Gellner, G., Moore, J.C., 2006. Structural asymmetry and the stability of diverse food webs. Nature 442, 265-269.

Schlesinger, W.H., 1997. Biogeochemistry. Academic Press, San Diego.

Sterner, R.W., Elser, J.J., 2002. Ecological Stoichiometry: The Biology of Elements from Molecules to the Biosphere. Princeton University Press, Princeton.

Watson, A.J., Lovelock, J.E., 1983. Biological homeostasis of the global environment - the parable of Daisyworld. Tellus B 35, 284-289.
Williams, R.J., Martinez, N.D., 2000. Simple rules yield complex food webs. Nature 404, 180-183.

Williams, R.J., Martinez, N.D., 2004. Stabilization of chaotic and nonpermanent food-web dynamics. Eur. Phys. J. B 38, 297-303.

Worm, B., Duffy, J.E., 2003. Biodiversity, productivity and stability in real food webs. Trends Ecol. Evol. 18, 628-632.

Wright, J.P., Jones, C.G., 2006. The concept of organisms as ecosystem engineers ten years on: progress, limitations, and challenges. Bioscience 56, 203-209.

Wright, J.P., Jones, C.G., Boeken, B., Shachak, M., 2006. Predictability of ecosystem engineering effects on species richness across environmental variability and spatial scales. J. Ecol. 94, 815-824.

Yodzis, P., 1981. The stability of real ecosystems. Nature 289, 674-676.

Yodzis, P., Innes, S., 1992. Body-size and consumer-resource dynamics. Am. Nat. 139, 1151-1173. 